\title{
Auto-stimulatory action of secreted caveolin-1 on the proliferation of Ewing's sarcoma cells
}

\author{
ANIRUDDHA SENGUPTA $^{1}$, SILVIA MATEO-LOZANO ${ }^{2}$, OSCAR M. TIRADO $^{3 *}$ and VICENTE NOTARIO ${ }^{*}$ \\ ${ }^{1}$ Department of Radiation Medicine, Lombardi Comprehensive Cancer Center, Georgetown University Medical Center, \\ Washington, DC, USA; ${ }^{2}$ Nanomedicine Research Program, Molecular Biology and Biochemistry Research Center, \\ CIBBIM-Nanomedicine, Vall d'Hebron Hospital Research Institute; ${ }^{3}$ Institut d'Investigació Biomèdica de Bellvitge \\ (IDIBELL), Laboratori d'Oncología Molecular, L'Hospitalet de Llobregat, Barcelona, Spain
}

Received January 25, 2011; Accepted February 21, 2011

DOI: $10.3892 /$ ijo.2011.963

\begin{abstract}
Caveolin-1 (CAV1) is highly expressed in Ewing's sarcoma (EWS). We previously showed that increased cellular CAV1 is associated with the regulation of the tumorigenicity, drug resistance and metastatic ability of EWS cells. Because several studies reported that melanoma and prostate cancer cells, which express relatively high CAV1 levels, secrete CAV1, and that secreted CAV1 is associated with tumor progression, our study explored the possibility that EWS cells also secreted CAV1 and that secreted CAV1 may contribute to EWS pathobiology. Results from experiments involving the ectopic expression of a Myc-tagged CAV1 protein in EWS cells as well as the supplementation of culture media with purified CAV1 protein followed by its intracellular localization using immunofluorescence demonstrated that EWS cells secrete CAV1, that they are able to take up the secreted protein, and that extracellular CAV1 enhances EWS cell proliferation. These findings strongly support the notion that secreted CAV1 may also contribute to the malignant properties of EWS.
\end{abstract}

\section{Introduction}

Ewing's sarcoma (EWS) is a highly malignant tumor of bones and soft tissues, primarily occurring in children and young adults (1). Most Ewing's sarcoma tumors are characterized by a $\mathrm{t}(11 ; 22)(\mathrm{q} 24 ; \mathrm{q} 12)$ chromosomal translocation (2), which generates the chimeric EWS/FLI protein by fusion of the EWSR 1 gene on chromosome 22 and the FLII gene on chromosome $11(3)$. This chimeric protein is an aberrant transcription factor, responsible for the malignant properties of EWS and critical for their maintenance (3). In recent years,

Correspondence to: Dr Vicente Notario, Department of Radiation Medicine, Lombardi Comprehensive Cancer Center, Georgetown University Medical Center, Washington, DC 20057, USA

E-mail: notariov@georgetown.edu

*Co-senior authors

Key words: caveolin-1, cell proliferation, Ewing's sarcoma, protein secretion, protein uptake therapeutic approaches have increased survival rates in EWS patients to about $60 \%$ (4), with surgery and radiotherapy being the major tools. However, the poor prognosis of EWS along with concerns over the effects of radiation led to the initiation of research efforts for the development of new therapeutic agents. Identification of proteins that may play a role in determining the sensitivity or resistance of EWS cells to chemotherapy may improve EWS treatment and patient survival. Genes transcriptionally regulated by EWS/FLI1 have been cited often as important mediators of oncogenesis, suggesting that targeting them may improve EWS treatment. A number of EWS/FLI1 transcriptional targets, with varied functions, have been identified (5). These proteins themselves or their interactions with other cellular proteins (6) could be potential therapeutic targets.

In an attempt to detect novel therapeutic targets, we identified the gene encoding caveolin-1 (CAVI) as a downstream direct transcriptional target of EWS/FLI1, with a role in controlling the malignant phenotype and tumorigenicity of EWS cells (7). The CAV1 protein, an important component of caveolae, plays major roles in cellular processes such as lipid transport, membrane trafficking, gene regulation, cell adhesion and signal transduction (8-10). CAV1 has been reported to contribute to malignancy through several mechanisms (11-13), including elevated expression as reported for meningioma (14), lung (15), renal (16), esophageal (17) and prostate (18) cancers. In addition, we have shown that the expression levels of CAV1 are inversely related to the sensitivity of EWS cells to chemotherapeutic agents (19). Knocking CAV1 down increased the sensitivity of EWS cells to clinically relevant agents such as doxorubicin and cisplatin, with a concurrent induction of apoptosis (19). Most recently, it has been demonstrated that CAV1 plays a key role in regulating the metastatic ability of EWS cells (20).

Several studies have established that in addition to its intracellular localization, CAV1 is also secreted through various mechanisms. One of the earlier works in this field suggested that CAV1 was secreted by exocrine cells and that its secretion was regulated by the exocrine pathway (21), as results showed that CAV1 was released from pancreas upon stimulation by agents known to enhance secretion from exocrine cells (21). Different functions have been attributed to secreted CAV1. In 
this regard, CAV1 secreted from osteoblasts influenced osteoblast calcification (22), promoted anchorage independence, migration and invasion in aggressive melanoma lines (23) and was elevated in plasma of melanoma patients (24). Elevated CAV1 secretion has been detected in parallel to the increased aggressiveness of prostate cancer cell lines, and the secreted CAV1 has been associated with proangiogenic activities and stimulation of proliferation and progression of prostate cancer (25-28). In light of the secretory nature of CAV1 and its consequences, the current study was undertaken to investigate the possible secretion of CAV1 by EWS cells and its cellular significance. Our observations indicate that CAV1 is secreted into the culture media by EWS cells, and that the secreted CAV1 is taken up by the same or adjacent cells and is capable of enhancing EWS cell proliferation.

\section{Materials and methods}

Reagents. DMEM, trypsin and antibiotics were purchased from Mediatech Inc. (Manassas, VA, USA). Antibodies against CAV1 and cell culture inserts with transparent polyethylene terephthalate (PET) membranes with 3- $\mu \mathrm{m}$ pores were from BD Biosciences (Franklin Lakes, NJ, USA). The antibody against the Myc tag and HRP-conjugated secondary antibodies were from Cell Signaling Technology (Danvers, MA, USA). Slide-A-Lyzer MINI Dialysis Units and the ECL Western blotting substrate were purchased from Thermo Scientific (Rockford, IL, USA). Lipofectamine 2000 and Alexa Fluor 488-conjugated secondary antibody were from Invitrogen (Carlsbad, CA, USA). The protease inhibitor cocktail was from Roche Applied Science (Indianapolis, IN, USA), the CellTiter-Glo Luminescent Cell Viability Assay kit was from Promega (Madison, WI, USA), the $0.45 \mu \mathrm{m}$ MCE filter units were from Fisher Scientific (Pittsburgh, PA, USA), and a TrueORF Gold Myc-DDK-tagged human CAVI plasmid was from Origene (Rockville, MD, USA). Anti-FLAG M2 Magnetic Beads were from Sigma-Aldrich (St. Louis, MO, USA) and Fluoro-Gel cell mounting medium was obtained from Electron Microscopy Sciences (Hatfield, PA, USA). Unless otherwise mentioned, most chemicals used in the study were of molecular biology or cell culture grade and were procured from either Fisher Scientific or Sigma-Aldrich.

Cell culture and preparation of conditioned media. The EWS cell lines (A4573, SK-ES-1 and TC-71) and the prostate cancer cell line (PC-3) were maintained in DMEM basal medium supplemented with antibiotics and $10 \%$ fetal bovine serum, and is henceforth referred to as the 'culture medium'. Cells were incubated at $37^{\circ} \mathrm{C}$ in a humidified atmosphere with $5 \%$ $\mathrm{CO}_{2}$. The CAV1 knocked-down A4573/shCAV1 cells were generated and maintained as described earlier (7). Conditioned media were prepared by collecting the culture fluids from sub-confluent cultures and clearing the cell debris by centrifugation at $1,000 \mathrm{xg}$, followed by filtration through $0.45 \mu \mathrm{m}$ MCE filter units.

Protein immunodetection. CAV1 from cell lysates or conditioned media was detected using either dot-blot assays or Western blotting. Cells were lysed with RIPA buffer containing protease inhibitor cocktail (Roche), and the lysates were centrifuged at
$13,000 \mathrm{x} \mathrm{g}$, at $4^{\circ} \mathrm{C}$, for $15 \mathrm{~min}$. Proteins obtained from lysed cells were resolved by 4-20\% SDS-PAGE, and transferred to PVDF membranes. To confirm the secretion of CAV1, different volumes of conditioned media from cultures of EWS cell lines and PC-3 cells were spotted on PVDF membranes, using a dot-blotting apparatus.

CAV1 immunodetection in media by Western blotting was carried out by concentrating the conditioned media through precipitation with either trichloroacetic acid (TCA) or ammonium sulfate. TCA (10\% by vol) was added to $1 \mathrm{ml}$ of conditioned media, incubated for $30 \mathrm{~min}$ on ice and centrifuged for $30 \mathrm{~min}$ at $10,000 \mathrm{x} \mathrm{g}$. The pellet was washed in acetone, air-dried and resuspended in $100 \mu \mathrm{l}$ of SDS sample buffer. Proteins present in the pellet (30 $\mu \mathrm{l})$ were resolved by $4-20 \%$ SDS-PAGE, and transferred to PVDF membranes.

For ammonium sulfate precipitation of proteins, conditioned media were centrifuged at $1,000 \mathrm{x} \mathrm{g}$ for $15 \mathrm{~min}$, at $4^{\circ} \mathrm{C}$, to remove debris. The supernatant collected was subjected to sequential $0-30,30-70$ and $70-90 \%$ ammonium sulfate fractionation, at $4^{\circ} \mathrm{C}$, maintaining the $\mathrm{pH}$ between 7.2 and 7.6. The ammonium sulfate precipitated fractions were resuspended in 150-200 $\mu \mathrm{l}$ of PBS and dialyzed in Slide-A-Lyzer MINI Dialysis Units against 11 of PBS for $16 \mathrm{~h}$. The dialyzed samples were centrifuged at $10,000 \mathrm{xg}$, at $4^{\circ} \mathrm{C}$, for $30 \mathrm{~min}$, to remove any particulate matter, and the concentration of protein was measured. Equal amounts of protein were subsequently subjected to SDS/PAGE analysis to optimize the percentage of ammonium sulfate required for CAV1 precipitation from the media.

Following transfer, the PVDF membranes were blocked with 5\% skim milk in PBST (PBS containing 0.2\% Tween-20) at room temperature for $1 \mathrm{~h}$ and incubated overnight at $4^{\circ} \mathrm{C}$ with designated primary antibodies, with gentle rocking. Next, the blots were washed with PBST and incubated at room temperature for $1 \mathrm{~h}$ with an HRP-conjugated secondary antibody and peroxidase activity was detected by enhanced chemiluminescence, using the ECL Western blotting substrate.

Transfection of human CAVI. To express CAV1 in EWS cells, a validated TrueORF Gold Myc-DDK-tagged human $C A V I$ plasmid was transfected into EWS cells using Lipofectamine 2000 following the manufacturer's protocols. Transfected cells were selected with G418 (1 mg/ml) for 14 days, and antibioticresistant colonies were pooled for further analysis and maintained in the presence of G418 $(0.5 \mathrm{mg} / \mathrm{ml})$.

Purification of Myc-DDK-CAVI. The DDK tag is a synonym for FLAG tag, hence Myc-DDK-tagged CAV1 expressed in A4573 and SK-ES-1 cells was purified using anti-FLAG magnetic beads according to the manufacturer's protocol. Briefly, cells expressing the tagged protein were lysed at $4^{\circ} \mathrm{C}$ for $15 \mathrm{~min}$ in lysis buffer containing $50 \mathrm{mM}$ Tris-Cl, $\mathrm{pH} 7.4$, $150 \mathrm{mM} \mathrm{NaCl}, 1 \mathrm{mMEDTA}$, and $1 \%$ Triton X-100, supplemented with the protease inhibitor cocktail. Lysates were centrifuged at $13,000 \times \mathrm{g}$, at $4^{\circ} \mathrm{C}$, for $15 \mathrm{~min}$. About $300-400 \mu 1$ of cell lysate were added to $40 \mu \mathrm{l}$ of resin beads, and the volume was made up to $1 \mathrm{ml}$ with the lysis buffer. The sample was incubated at room temperature on a roller shaker for $2 \mathrm{~h}$ and then placed on a magnetic separator to remove the supernatant. The beads were washed 5 times with TBS (50 mM Tris- $\mathrm{Cl}$, 
$150 \mathrm{mM} \mathrm{NaCl}, \mathrm{pH} 7.4$ ), and bound protein was eluted with $50 \mu \mathrm{l}$ of $0.1 \mathrm{M}$ glycine $\mathrm{HCl}, \mathrm{pH} 3.0$. The eluted protein was neutralized with $15 \mu \mathrm{l}$ of Tris- $\mathrm{Cl}, \mathrm{pH} 8.0$, and subsequently used for further studies. The Myc-DDK-tagged CAV1 is henceforth referred to as MD-CAV1.

Immunofluorescence studies. A4573 cells were seeded on glass cover slips in 12-well plates and grown in $1 \mathrm{ml}$ culture medium for 2 days. The culture medium was replaced with $400 \mu \mathrm{l}$ of fresh medium in each well. Subsequently, three different sets were assigned for experimental purposes. In the first set, cell culture inserts with transparent PET membranes (with $3-\mu \mathrm{m}$ pores) were added to wells with cells on cover slips and $500 \mu \mathrm{l}$ of culture-media containing $2 \times 10^{4}$ A4573 cells expressing MD-CAV1 were seeded on the membrane in the inserts. In the second set, purified MD-CAV1 was added in $500 \mu \mathrm{l}$ of culture medium directly to the cells on cover slips, while in the third set fresh culture medium was added to the cells on cover slips. The cells were allowed to grow for $16 \mathrm{~h}$, following which the cells on the cover slips were fixed in $4 \%$ paraformaldehyde, permeabilized with $0.1 \%$ Triton X-100 and equilibrated in PBS. The cover slips with fixed cells were pre-incubated for $30 \mathrm{~min}$ in $2 \%$ BSA-PBS, followed by $1-\mathrm{h}$ incubation with mouse anti-Myc antibody (1:5000 dilution in $1 \%$ BSA-PBS) at room temperature. The cover slips were washed thrice with PBS and incubated for 45 min with Alexa Fluor 488 conjugated secondary antibody (1:2000 dilutions in $1 \%$ BSA-PBS). The cells were washed twice with PBS, before incubating them with DAPI for $30 \mathrm{~min}$ in the dark. Finally, the cells were washed twice in PBS and once in deionized water before being mounted in Fluoro-Gel mounting medium. Images were recorded in an Olympus microscope and analyzed using software provided by the manufacturer.

Cell proliferation assays. Cell proliferation was measured using the CellTiter-Glo Luminescent Cell Viability Assay kit, according to the manufacturer's protocol in a 96-well plate format. Equal number of cells were seeded in 96-well plates and following $24 \mathrm{~h}$ of growth were subjected to the following conditions: i) culture-medium (control); ii) 1:1 CAV1 conditioned medium and culture-medium; iii) culture medium with purified MD-CAV1; iv) culture-medium with purified MD-CAV1 plus anti-CAV1 antibodies; and v) culture-medium with anti-CAV1 antibodies only. Following growth for $48 \mathrm{~h}$, the luminescence intensity measured for each sample was plotted relative to the values obtained for the control cells. The data represent the average from at least 3 independent experiments.

Statistical analysis. Significance (p)-values between dataset pairs were obtained from two-tailed Student's t-tests, and the data presented as the average with the standard deviation as error bars.

\section{Results}

Secretion of CAV1 by EWS cells. To evaluate if CAV1 was secreted by EWS cells, 300 and $100 \mu \mathrm{l}$ of unconcentrated conditioned media collected from exponentially growing cultures of EWS lines and PC-3 cells were spotted on PVDF membranes using a dot-blot apparatus, and subjected to immuno-
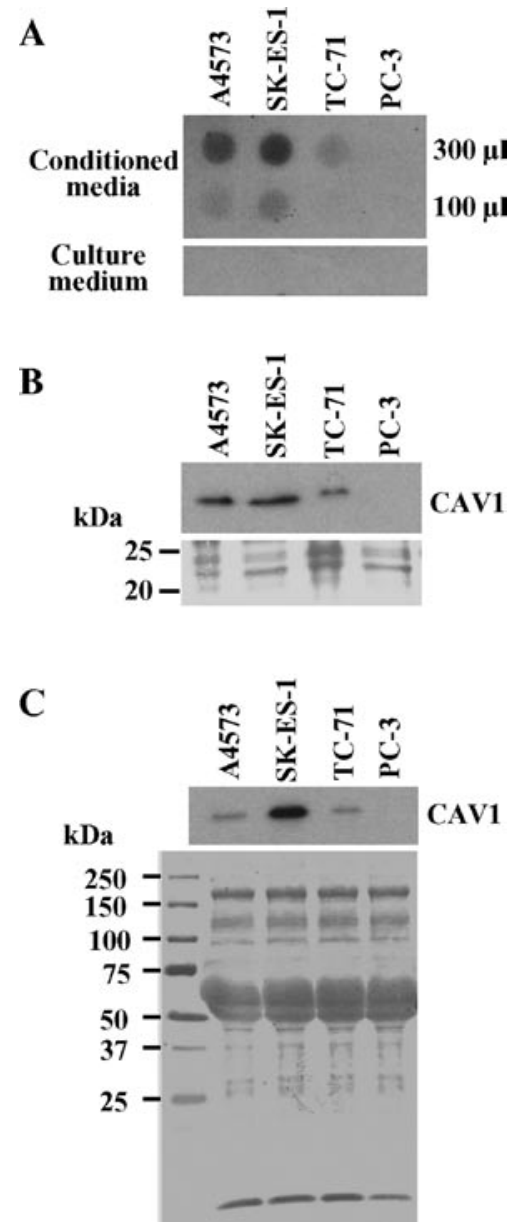

Figure 1. Secretion of CAV1 by EWS cells. (A) Detection of CAV1 with anti-CAV1 antibodies through dot-blot analysis of the indicated volumes of unconcentrated conditioned media from cultures of A4573, SK-ES-1, TC-71 and PC-3 cells (upper panel); fresh culture medium was used as control (lower panel). (B) Immunodetection of CAV1 in TCA precipitates obtained from conditioned media prepared from cultures of the same cell lines, and resolved by SDS-PAGE (upper panel); Ponceau staining of the region of the membrane corresponding to the range of the CAV1 molecular mass (lower panel) is shown as loading reference. (C) Western blot analysis for CAV1 in the protein fraction purified from conditioned media from EWS and PC-3 cells by $30-70 \%$ ammonium sulfate precipitation, and resolved on SDS-PAGE (upper panel); Ponceau staining of the blot is shown as control for equal loading (lower panel). A short exposure of the immunoblots is shown in panels (B) and (C) to better illustrate expression differences.

detection using anti-CAV1 antibodies. Results indicated that CAV1 was secreted by the three EWS cell lines tested, while no signal was detected in the case of PC-3 cells under the same experimental conditions (Fig. 1A). As expected, culture medium alone, used as negative control, did not yield any signal. After this initial finding of CAV1 in the culture fluids of EWS cells, conditioned media collected from the EWS lines as well as from PC-3 cells was subjected to TCA precipitation, and proteins present in the precipitates were resolved by SDS-PAGE, blotted onto PVDF membranes and subjected to immunodetection with anti-CAV1 antibodies. Results indicated that EWS cells secrete CAV1 in variable amounts (Fig. 1B), whereas no detectable CAV1 immunoreactivity was observed in case of the PC-3 cells unless the immunoblot was subjected to much longer exposures. Proteins present in the conditioned media prepared from EWS cell cultures were fractionated by 0-30, 30-70 and 
A

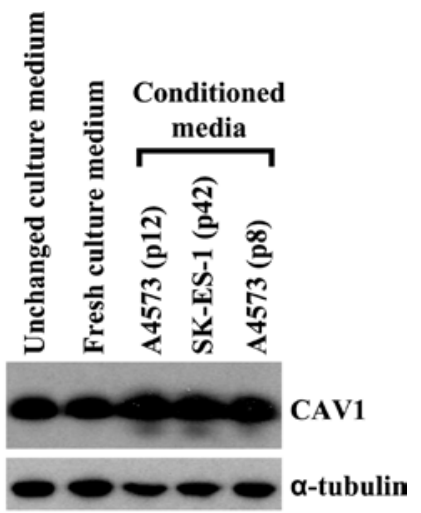

B

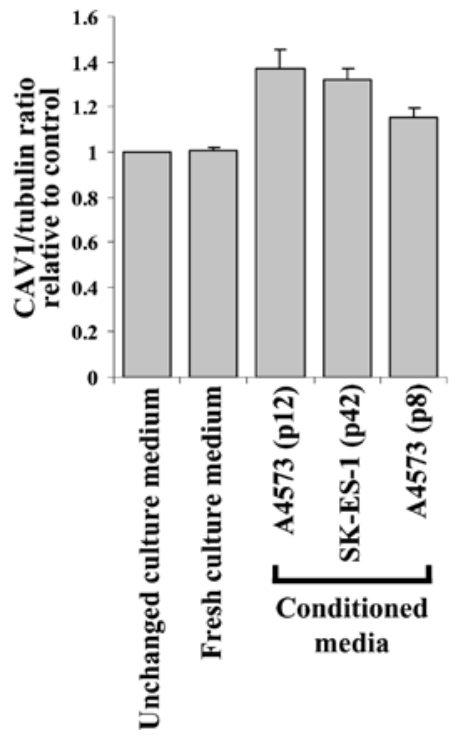

Figure 2. Secreted CAV1 is taken up by EWS cells. (A) Detection of cellular CAV1 protein levels in CAV1 knocked-down A4573/shCav1 cells, grown in the presence and absence of conditioned media prepared from the EWS cell lines and passages indicated (upper panel); $\alpha$-tubulin (lower panel) was used as the loading control. (B) Quantification of the relative differences in cellular CAV1 content under the experimental conditions indicated.

70-90\% ammonium sulfate precipitation, and subsequently resolved on SDS-PAGE and subjected to immunodetection with anti-CAV1 antibodies. Secreted CAV1 was detected in the $30-70 \%$ ammonium sulfate fraction (Fig. 1C), while no CAV1 immunoreactivity was detected in any of the other fractions (data not shown). Taken together, these data clearly demonstrate the secretion of CAV1 by EWS cells.

Secreted CAV1 is taken up by EWS cells. To explore the possibility that secreted CAV1 may be taken up by EWS cells, we used A4573/shCav1 cells, which were engineered to express diminished levels of CAV1 (7). In these cells, CAV1 uptake could result in increased endogenous CAV1 levels, and such an increase could be detected by CAV1 immunodetection in total cell lysates. A4573/shCavl cells were cultured in regular culture medium for 2 days, and then further allowed to grow: i) in the same unchanged culture medium, ii) in fresh culture medium, or iii) in a 1:1 mix of culture medium and conditioned media from A4573 cells (prepared from two different culture passages) or from SK-ES-1 cells. After $48 \mathrm{~h}$ under these
A

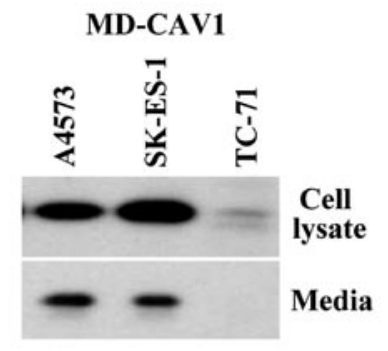

B

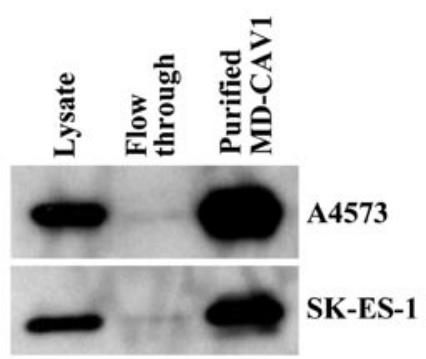

C

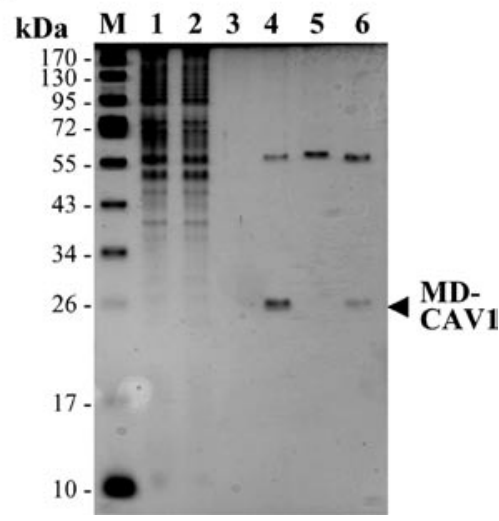

Figure 3. Ectopically expressed human CAV1 is secreted and taken up by EWS cells. (A) The Myc-DDK-tagged (MD-CAV1) protein was detected using anti-Myc antibodies in cell lysates (upper panel) and conditioned media (lower panel) prepared from the indicated EWS cells stably transfected with Myc-DDK-CAV1. (B) The MD-CAV1 protein was purified from A4573 (upper panel) and SK-ES-1 cells (lower panel) using anti-FLAG magnetic beads; samples of the cell lysates, flow through and the eluted fractions were resolved by SDS-PAGE and subjected to immunodetection with anti-Myc antibodies. (C) Purification of MD-CAV1. MD-CAV1 was purified using anti-FLAG magnetic beads as described in Materials and methods. Samples from the various steps of the purification process were resolved by SDS-PAGE, and the purity of the protein was assessed using silver staining. M, denotes marker, while lanes correspond to the following: (1) cell lysate, $3 \mu 1$; (2) flow through following incubation with anti-FLAG beads, $3 \mu$; (3) TBS washing of the beads, $3 \mu \mathrm{l}$; (4) bound protein eluted with glycine $\mathrm{HCl}, 10 \mu \mathrm{l}$; (5) magnetic beads alone treated with glycine $\mathrm{HCl}, 10 \mu \mathrm{l}$; and (6) bound protein eluted with Laemmli buffer, $10 \mu 1$.

conditions, the CAV1 knocked-down cells were harvested, lysed and the lysates subjected to SDS-PAGE and CAV1 immunodetection with anti-CAV1 antibodies. Results indicated that, compared to the control cells growing in unchanged or fresh culture media, the cellular levels of CAV1 were elevated in all cases in which the A4573/shCavlcells were cultured with conditioned media prepared from the EWS cells (Fig. 2A). Densitometric quantification of the CAV1 levels showed that the endogenous CAV1 levels in A4573/shCav1 cells were increased up to $40 \%$ when cultured in the presence of conditioned media prepared from A4573 or SK-ES-1 (Fig. 2B). 


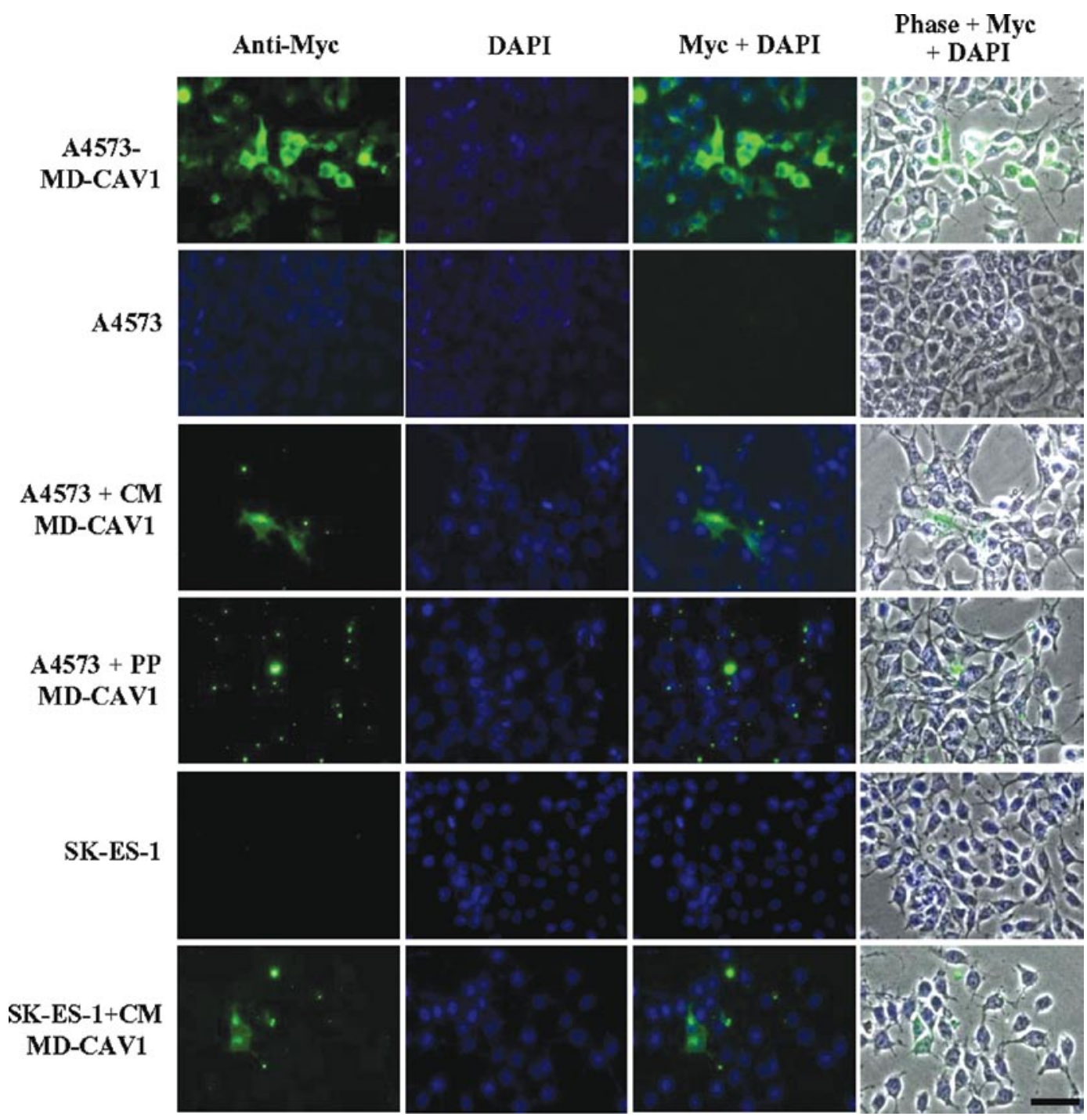

Figure 4. Uptake of human CAV1 by EWS cells. Immunofluorescent detection of CAV1 taken up by A4573 or SK-ES-1 cells either from conditioned medium (CM MD-CAV1) or from culture medium supplemented with the purified protein (PP MD-CAV1). MD-CAV1 was visualized using an Alexa Fluor 488 (green)-conjugated secondary antibody following immunodetection of the Myc tag, while the nuclei were visualized using DAPI (blue). The panels show individual channels detecting the protein (Anti-Myc) and nuclei (DAPI) along with fluorescent merged channels (Myc + DAPI) and fluorescent channels merged with phase contrast image (Phase + Myc + DAPI). The size bar corresponds to $50 \mu \mathrm{m}$.

Ectopically expressed human CAVI is secreted and taken up by EWS cells. In order to study the secretion of CAV1 and its possible uptake by EWS cells, human CAV1 tagged with the Myc-DDK epitopes was expressed in the A4573, SK-ES-1 and TC-71 EWS cell lines. Following transfection, stable EWS lines expressing MD-CAV1 were generated. Protein lysates and conditioned media from cells expressing MD-CAV1 were subjected to SDS-PAGE and Western blotting using anti-Myc antibodies for immunodetecting the tagged protein. Results showed different levels of the MD-CAV1 protein in total lysates of the three EWS cell lines, with TC-71 cells showing the lowest level of MD-CAV1 expression whereas the A4573 and SK-ES-1 cells expressed much greater MD-CAV1 levels (Fig. 3A, top panel). Interestingly, the MD-CAV1 protein was also detected in the conditioned media from both A4573 and SK-ES-1 cells (Fig. 3A, bottom panel), thus demonstrating the secretory nature of the tagged CAV1. Owing to its higher expression in A4573 and SK-ES-1 cells, MD-CAV1 was purified from both these cell lines using anti-FLAG magnetic beads, as described earlier (Fig. 3B). Samples of fractions from the purification process were resolved by SDS-PAGE and protein bands were visualized with silver staining to determine the purity of the protein (Fig. 3C). Following the detection of expressed MD-CAV1 in the culture fluids of EWS cells, we investigated the possible uptake of the ectopically expressed, tagged CAV1 by A4573 cells. A4573 cells were seeded on sterile cover slips and grown for 2 days; then they were incubated in the presence of either MD-CAV1-containing condition medium or with purified MD-CAV1 protein $(0.3 \mu \mathrm{g} / \mathrm{ml})$, as detailed in Materials and methods. After $16 \mathrm{~h}$ in culture, the cells were fixed and subjected to immunofluorescence analysis to detect any MD-CAV1 protein taken up by the A4573 cells. Immunofluorescence data indicated that the A4573 cells did take up the MD-CAV1 protein both from MD-CAV1-containing conditioned medium and from culture medium supplemented with purified protein (Fig. 4). MD-CAV1 taken up by the cells was visualized as green punctuate dots through detection of the Myc tag, while the nuclei were stained with DAPI. A 
A

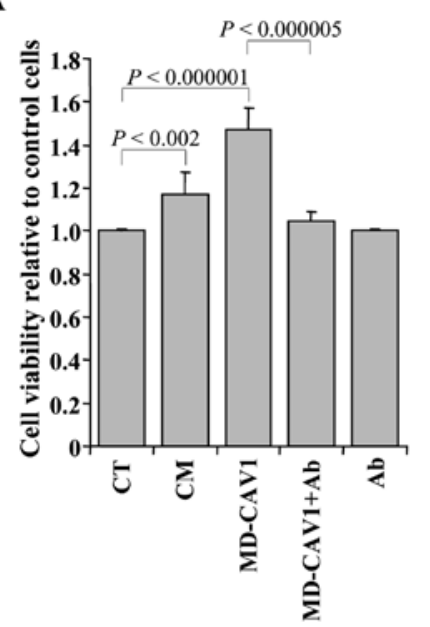

B

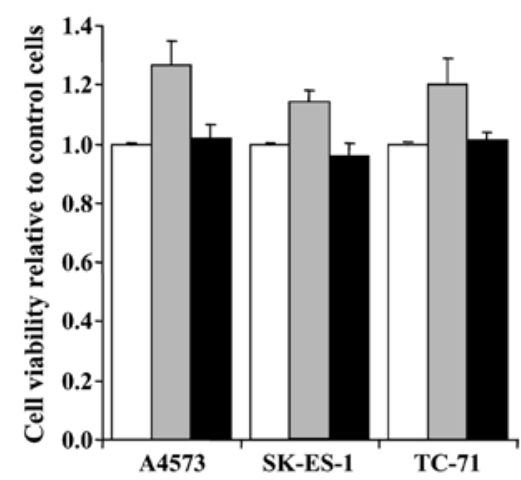

Figure 5. Exogenously added CAV1 stimulates cell proliferation in EWS cells. (A) Proliferation of A4573 cells was measured using a luminescencebased technique in untreated cells (CT), and in cells incubated with conditioned medium containing CAV1 (CM); with exogenously added purified MD-CAV1; with exogenously added purified MD-CAV1 along with anti-CAV1 antibody (MD-CAV1 + Ab); and with the anti-CAV1 antibody alone (Ab). (B) Comparison of proliferation of EWS cell lines in the presence of either exogenously added MD-CAV1 (gray bar) or exogenously added MD-CAV1 plus the anti-CAV1 polyclonal antibody (black bar), with the control cells (white bar). Cell viability values in both panels are plotted relative to those in untreated control cultures, which were arbitrarily set as 1.00 .

similar uptake pattern was noted in SK-ES-1 cells (Fig. 4). These observations demonstrate that CAV1 is secreted into the media by EWS cells and that the secreted protein may also be taken up by the same or adjacent cells.

Exogenously added CAVI stimulates cell proliferation in EWS cells. The possible role of secreted CAV1 in EWS cells was explored by investigating the effects of exogenously added CAV1 or CAV1-containing conditioned medium on the proliferation of A5473 cells. Either purified MD-CAV1 protein $(0.9 \mu \mathrm{g} / \mathrm{ml})$ or CAV1-containing conditioned medium prepared from cultured A4573 cells were added to A4573 cells after $24 \mathrm{~h}$ in culture, and cell proliferation was measured $48 \mathrm{~h}$ later using a luminescence-based technique. Results indicated that, in comparison to control cells maintained in regular culture medium, both purified MD-CAV1 $(\mathrm{p}<0.000001)$ and CAV1containing medium $(\mathrm{p}<0.002)$ were capable of promoting statistically significant increases in cell proliferation (Fig. 5A). To determine whether this effect was induced by CAV1 rather than any other compound present in the conditioned medium or as a minor contaminant in the preparation of purified MD-CAV1, A4573 cells were incubated with purified MD-CAV1 protein that had been pre-incubated with polyclonal anti-CAV1 antibody. Blocking the purified MD-CAV1 protein with anti-CAV1 antibody significantly $(\mathrm{p}<0.000005)$ reduced its stimulatory effect on cell proliferation (Fig. 5A). To confirm that the stimulatory activity of exogenous CAV1 on cell proliferation was not just a peculiar response of the A4573 cells, we examined the effect of purified MD-CAV1 on all the three EWS cell lines used in this study, in the presence or absence of the polyclonal anti-CAV1 antibody. Although the extent of the stimulatory effect was different for the three cell lines, the results showed similar patterns (Fig. 5B). Exogenously added MD-CAV1 caused a stimulation of the proliferation of the EWS cells that was blocked when CAV1 was pre-incubated with anti-CAV1 antibody. Overall, these data indicate that secreted CAV1 may participate in a self-stimulatory loop regulating the proliferation of EWS cells.

\section{Discussion}

The complexity and poor prognosis of EWS calls for the identification of new targets for therapeutic purposes. EWS/ FLI1 is a key protein in EWS pathobiology, and its transcriptional targets are often considered as putative therapeutic targets. This provides impetus for the identification and molecular analysis of genes/protein transcriptionally regulated by EWS/FLI1, as a source of mechanistic insight into the proliferation and malignant properties of this aggressive sarcoma. In this context, we identified CAV1 as an EWS/FLI1 direct transcriptional target, and showed its involvement in the regulation of the malignant phenotype and tumorigenicity of EWS cells (7). In a subsequent study, we have shown that the expression levels of CAV1 are related to the resistance of EWS cells to chemotherapeutic agents, as a decrease in CAV1 concentration sensitizes EWS cells to drug treatment (19). Most recently, we also showed that CAV1 is a key regulator of the ability of EWS cells to metastasize (20). However, most of these cellular processes and activities have been related to the endogenous cellular levels of CAV1, but it was not known whether EWS cells secreted CAV1 and the possible significance of any secreted CAV1 form for EWS pathophysiology.

The current study represents the first report on the secretory nature of CAV1 in EWS cells and its involvement in regulating cell proliferation. Significant levels of CAV1 were detected in conditioned media from EWS cells (A4573, SK-ES-1, TC-71), while nearly undetectable levels were present in the conditioned media of prostate cancer PC-3 cells, suggesting an elevated secretion of CAV1 by EWS cells (Fig. 1). Interestingly, there does not seem to be a correlation between the total endogenous levels of CAV1 present in the three EWS cell lines and the relative levels of CAV1 that they secrete, as A4573 cells contain the highest levels of cellular CAV1 protein (7), whereas the largest amounts of secreted CAV1 were found in conditioned media from SK-ES-1 cell cultures. Culturing A4573/shCav1 cells, which were engineered to express diminished levels of CAV1 by shRNA-mediated CAV1 knockdown (7), with conditioned medium prepared from A4573 cell culture fluids resulted in an increase in the levels of intracellular CAV1, strongly suggesting that the secreted protein was taken 
up by the A4573/shCav1 cells (Fig. 2). To confirm the secretory nature of CAV1 in EWS cells and to investigate its possible significance for EWS pathophysiology, we ectopically expressed a human Myc-DDK-tagged CAV1 in EWS cells. Following the generation of stably transfected cell lines, the tagged MD-CAV1 protein was detected in the conditioned media from EWS cell lines (Fig. 3A). The fact that MD-CAV1 was secreted by these cells in a manner similar to the secretion of endogenous CAV1, as previously observed (Fig. 1), with SK-ES-1 cells secreting the highest levels in both cases, suggested that the endogenous and the tagged CAV1 proteins were likely processed in EWS cells via the same secretory pathways. Subsequent immunofluorescence experiments revealed that MD-CAV1, both as component of conditioned media or as a purified protein, was taken up by A4573 cells (Fig. 4). The functional significance of the secreted protein was examined by testing the effects of the addition of either purified MD-CAV1 protein or MD-CAV1-containing conditioned media on the proliferation of EWS cells. Results indicated that the proliferation in A4573 cells was specifically enhanced by the secreted MD-CAV1 protein, as its effect on cell proliferation was prevented by pre-incubation of the purified protein with anti-CAV1 polyclonal antibodies (Fig. 5A). Though the magnitude of the enhancing effect of secreted CAV1 on cell proliferation was different in the three EWS cell lines tested, the fact that a stimulatory response was observed in all cases suggests that secreted CAV1 may be a positive effector of cell proliferation for EWS cells in general (Fig. 5B).

Although additional studies are needed to characterize the mechanism by which CAV1 enters the secretory pathway and other pathways influenced by it, as well as the mechanisms by which CAV1 is taken up by EWS cells, the current study clearly shows that CAV1 is secreted by EWS cells, that EWS cells are able to take up the secreted protein, and that secreted CAV1 enhances the proliferation of EWS cells. These findings strongly support the notion that secreted CAV1 may also contribute to the malignant properties of EWS. In light of these results, it seems possible that secreted CAV1 may promote the growth of metastases in vivo, and this additional molecular insight raises even further the possibility of using CAV1 as a therapeutic target for Ewing's sarcoma.

\section{Acknowledgements}

This work was supported by the US National Cancer Institute, USPHS grant RO1-CA134727 (to V.N.), by the 'Fondo de Investigaciones Sanitarias-ISCIII' (CP06/00151; PI080259, to O.M.T.), and by the Tissue Culture and the Microscopy and Imaging Shared Resources of the Lombardi Comprehensive Cancer Center that are funded through USPHS grant P30-CA-CA51008.

\section{References}

1. Arndt CA and Crist WM: Common musculoskeletal tumors of childhood and adolescence. N Engl J Med 341: 342-352, 1999.

2. Turc-Carel C, Aurias A, Mugneret F, et al: Chromosomes in Ewing's sarcoma. I. An evaluation of 85 cases of remarkable consistency of $\mathrm{t}(11 ; 22)(\mathrm{q} 24 ; \mathrm{q} 12)$. Cancer Genet Cytogenet 32: 229-238, 1988

3. Delattre O, Zucman J, Plougastel B, et al: Gene fusion with an ETS DNA-binding domain caused by chromosome translocation in human tumours. Nature 359: 162-165, 1992.
4. Linabery AM and Ross JA: Childhood and adolescent cancer survival in the US by race and ethnicity for the diagnostic period 1975-1999. Cancer 113: 2575-2596, 2008.

5. Toomey EC, Schiffman JD and Lessnick SL: Recent advances in the molecular pathogenesis of Ewing's sarcoma. Oncogene 29: 4504-4516, 2010

6. Erkizan HV, Uversky VN and Toretsky JA: Oncogenic partnerships: EWS-FLI1 protein interactions initiate key pathways of Ewing's sarcoma. Clin Cancer Res 16: 4077-4083, 2010.

7. Tirado OM, Mateo-Lozano S, Villar J, et al: Caveolin-1 (CAV1) is a target of EWS/FLI-1 and a key determinant of the oncogenic phenotype and tumorigenicity of Ewing's sarcoma cells. Cancer Res 66: 9937-9947, 2006.

8. Williams TM and Lisanti MP: Caveolin-1 in oncogenic transformation, cancer, and metastasis. Am J Physiol Cell Physiol 288: 494-506, 2005.

9. Shaul PW and Anderson RG: Role of plasmalemmal caveolae in signal transduction. Am J Physiol 275: 843-851, 1998.

10. Sternberg PW and Schmid SL: Caveolin, cholesterol and Ras signaling. Nat Cell Biol 1: 35-37, 1999.

11. Cavallo-Medved D, Mai J, Dosescu J, Sameni M and Sloane BF: Caveolin-1 mediates the expression and localization of cathepsin $\mathrm{B}$, pro-urokinase plasminogen activator and their cell-surface receptors in human colorectal carcinoma cells. J Cell Sci 118: 1493-1503, 2005.

12. Li L, Yang G, Ebara S, et al: Caveolin-1 mediates testosteronestimulated survival/clonal growth and promotes metastatic activities in prostate cancer cells. Cancer Res 61: 4386-4392, 2001.

13. Woodman SE, Ashton AW, Schubert W, et al: Caveolin-1 knockout mice show an impaired angiogenic response to exogenous stimuli. Am J Pathol 162: 2059-2068, 2003.

14. Barresi V, Cerasoli S, Paioli G, et al: Caveolin-1 in meningiomas: expression and clinico-pathological correlations. Acta Neuropathol 112: 617-626, 2006.

15. Ho CC, Huang PH, Huang HY, Chen YH, Yang PC and Hsu SM: Up-regulated caveolin-1 accentuates the metastasis capability of lung adenocarcinoma by inducing filopodia formation. Am J Pathol 161: 1647-1656, 2002.

16. Joo HJ, Oh DK, Kim YS, Lee KB and Kim SJ: Increased expression of caveolin-1 and microvessel density correlates with metastasis and poor prognosis in clear cell renal cell carcinoma. BJU Int 93: 291-296, 2004

17. Kato K, Hida Y, Miyamoto M, et al: Overexpression of caveolin-1 in esophageal squamous cell carcinoma correlates with lymph node metastasis and pathologic stage. Cancer 94: 929-933, 2002.

18. Yang G, Truong LD, Timme TL, et al: Elevated expression of caveolin is associated with prostate and breast cancer. Clin Cancer Res 4: 1873-1880, 1998.

19. Tirado OM, MacCarthy CM, Fatima N, Villar J, Mateo-Lozano S and Notario V: Caveolin-1 promotes resistance to chemotherapyinduced apoptosis in Ewing's sarcoma cells by modulating PKCalpha phosphorylation. Int J Cancer 126: 426-436, 2010.

20. Sáinz-Jaspeado M, Lagares-Tena L, Lasheras J, et al: Caveolin-1 modulates the ability of Ewing's sarcoma to metastasize. Mol Cancer Res 8: 1489-1500, 2010.

21. Liu P, Li WP, Machleidt T and Anderson RG: Identification of caveolin-1 in lipoprotein particles secreted by exocrine cells. Nat Cell Biol 1: 369-375, 1999.

22. Sawada N, Taketani Y, Amizuka N, et al: Caveolin-1 in extracellular matrix vesicles secreted from osteoblasts. Bone 41: 52-58, 2007.

23. Felicetti F, Parolini I, Bottero L, et al: Caveolin-1 tumor-promoting role in human melanoma. Int J Cancer 125: 1514-1522, 2009.

24. Logozzi M, De Milito A, Lugini L, et al: High levels of exosomes expressing CD63 and caveolin-1 in plasma of melanoma patients. PLoS One 4: e5219, 2009.

25. Tahir SA, Yang G, Ebara S, et al: Secreted caveolin-1 stimulates cell survival/clonal growth and contributes to metastasis in androgen-insensitive prostate cancer. Cancer Res 61: 3882-3885, 2001.

26. Tahir SA, Yang G, Goltsov AA, et al: Tumor cell-secreted caveolin-1 has proangiogenic activities in prostate cancer. Cancer Res 68: 731-739, 2008.

27. Bartz R, Zhou J, Hsieh JT, Ying Y, Li W and Liu P: Caveolin-1 secreting LNCaP cells induce tumor growth of caveolin-1 negative LNCaP cells in vivo. Int J Cancer 122: 520-525, 2008.

28. Watanabe M, Yang G, Cao G, et al: Functional analysis of secreted caveolin-1 in mouse models of prostate cancer progression. Mol Cancer Res 7: 1446-1455, 2009. 